\title{
TITLE:
}

\section{A note on regularity conditions on ideal magnetohydrodynamic equations}

\author{
$\operatorname{AUTHOR}(\mathrm{S})$ :
}

Ohkitani, K

\section{CITATION:}

Ohkitani, K. A note on regularity conditions on ideal

magnetohydrodynamic equations. PHYSICS OF PLASMAS 2006, 13(4): 044504.

\section{ISSUE DATE:}

2006-04

URL:

http://hdl.handle.net/2433/50502

\section{RIGHT:}

Copyright 2006 American Institute of Physics. This article may be downloaded for personal use only. Any other use requires prior permission of the author and the American Institute of Physics. 


\title{
A note on regularity conditions on ideal magnetohydrodynamic equations
}

\author{
K. Ohkitani \\ Research Institute for Mathematical Sciences, Kyoto University, Kyoto 606-8502, Japan
}

(Received 22 February 2006; accepted 24 March 2006; published online 21 April 2006)

\begin{abstract}
A regularity criterion for ideal magnetohydrodynamical equations is known to be given in terms of the vorticity and the current density fields [Caflisch et al., Commun. Math. Phys. 184, 443 (1997)]. A simple argument which utilizes Weber transform associated with cross helicity invariant shows that if the magnetic field is smooth, then so is the velocity field, thereby suggesting some room for improving the above criterion. (C) 2006 American Institute of Physics. [DOI: 10.1063/1.2196242]
\end{abstract}

One of the longstanding problems of ideal, incompressible fluid motion is whether it leads to formation of singularities in finite time. There are many mathematical and numerical works which study the possibility of such singularities for 3D Euler equations. However, so far no conclusive result has been given analytically as to their presence or absence.

As for ideal magnetohydrodynamic equations, at present we have no mathematical theory which guarantees the existence of solutions to the system either. Some works report numerical simulations suggesting singularity formation in finite time, for example, Ref. 1 . There are some relevant theoretical studies, ${ }^{2,3}$ but in general cases, singularity formation in magnetohydrodynamics remains an open problem. In the magnetohydrodynamic cases, a satisfactory mathematical theory is lacking to rule out singularity formation even in two dimensions. In the studies, ${ }^{4,5}$ some flow patterns observed in numerics are assumed and discuss regularity for the restricted class of flows. The study of singularity formation in magnetohydrodynamics is important not only mathematically, but also physically because singularity, if present, can cause magnetic reconnection.

The purpose of this Brief Communication is to remark about regularity conditions for ideal magnetohydrodynamic equations. We pay attention to a correspondence between the Eulerian and the Lagrangian representations.

With standard notations, the 3D Euler equations for the velocity $\boldsymbol{u}=(u, v)$ satisfy

$$
\begin{aligned}
& \frac{D \boldsymbol{u}}{D t}=-\nabla p, \\
& \nabla \cdot \boldsymbol{u}=0,
\end{aligned}
$$

where $p$ is the pressure. It is well-known that for flows starting from smooth initial conditions, as long as

$$
\int_{0}^{T} \sup _{x}|\boldsymbol{\omega}| d t<\infty,
$$

smoothness is guaranteed on the time interval $0 \leqslant t \leqslant T$ as proved by Ref. 6 . In other words, the vorticity alone controls regularity of solutions. On the other hand, the classical Cauchy formula states that

$$
\omega=\omega_{0} \cdot \frac{\partial}{\partial a} x(a, t)
$$

where $\boldsymbol{x}(\boldsymbol{a}, t)$ denotes a position at time $t$ of a fluid particle $\boldsymbol{a}$. We recall the Jacobian matrix $\partial x_{i} / \partial a_{j}$ dictates the transformation between the Eulerian and Lagrangian representations and its determinant is unity.

Combining with the above Beale-Kato-Majda criterion, we deduce that as long as the correspondence between the Eulerian and Lagrangian representations is nonsingular, smooth solutions persist for 3D Euler equations.

As another example we consider 2D Boussinesq equations,

$$
\begin{aligned}
& \frac{D \boldsymbol{u}}{D t}=-\nabla p+\left(\begin{array}{l}
0 \\
\theta
\end{array}\right), \\
& \frac{D \theta}{D t}=0, \\
& \nabla \cdot \boldsymbol{u}=0 .
\end{aligned}
$$

Unlike 2D Euler equations, scalar vorticity $\omega=\partial_{x} v-\partial_{y} u$ is not conserved for this system

$$
\frac{D \omega}{D t}=\frac{\partial \theta}{\partial x},
$$

and for this reason the regularity of Boussinesq equations is an open problem. A counterpart to the Beale-Kato-Majda criterion is given by

$$
\int_{0}^{T} \sup |\nabla \theta| d t<\infty,
$$

see, for example, Refs. 7 and 8. On the other hand, a counterpart to the Cauchy formula is the following first integral:

$$
\nabla^{\perp} \theta=\nabla^{\perp} \theta \cdot \frac{\partial}{\partial \boldsymbol{a}} \boldsymbol{x}(\boldsymbol{a}, t)
$$

which is just another way of stating that $\theta$ is conserved. This states that the skew gradient of temperature $\nabla^{\perp} \theta=\left(\partial_{y} \theta\right.$, $\left.-\partial_{x} \theta\right)$ is represented by components of the Jacobian matrix. Therefore, also in the case of 2D Boussinesq equations, we deduce that as long as the correspondence between the Eu- 
lerian and Lagrangian representations is nonsingular, smooth solutions persist.

We now consider the main system of 3D magnetohydrodynamic equations, which may be written as

$$
\begin{aligned}
& \frac{D \boldsymbol{u}}{D t}=-\nabla p+(\nabla \times \boldsymbol{B}) \times \boldsymbol{B}, \\
& \frac{D \boldsymbol{B}}{D t}=\boldsymbol{B} \cdot \nabla \boldsymbol{u}, \\
& \nabla \cdot \boldsymbol{u}=\nabla \cdot \boldsymbol{B}=0,
\end{aligned}
$$

where $\boldsymbol{B}$ denotes the magnetic field and $\boldsymbol{J}=\nabla \times \boldsymbol{B}$ is the current density. In Ref. 9, an analog of the Beale-Kato-Majda criterion has been established which states that, as long as

$$
\int_{0}^{T}\left(\sup _{\boldsymbol{x}}|\boldsymbol{\omega}|+\sup _{\boldsymbol{x}}|\boldsymbol{J}|\right) d t<\infty,
$$

smooth solutions persist.

The theorem has been proven by careful mathematical analyses after symmetrizing the 3D magnetohydrodynamic equations with use of Elsasser variables,

$$
z^{ \pm}=\boldsymbol{u} \pm \boldsymbol{B}
$$

Note that a curl of $z^{+}$suggests the integrand in the criterion in (1). A well-known analog of the Cauchy formula is

$$
\boldsymbol{B}=\boldsymbol{B}_{0} \cdot \frac{\partial}{\partial \boldsymbol{a}} \boldsymbol{x}(\boldsymbol{a}, t)
$$

which states that magnetic lines are frozen in fluids. It implies that as long as the magnetic field is regular, the correspondence between the Eulerian and Lagrangian representations is smooth.

In view of experiences with the two previous examples, we are tempted to infer that the magnetic field alone controls regularity. However, in Ref. 9, to ensure regularity, conditions are required both on the velocity (vorticity) field and the magnetic (current density) field.

A natural question is the following: is the magnetic field not sufficient for regularity of 3D magnetohydrodynamic equations? While at present we are not able to show a criterion like Beale-Kato-Majda's solely in terms of $\boldsymbol{B}$, we show by taking into account conservation laws appropriately that if the magnetic field is smooth, so is the velocity field. The essential point in the argument is utilization of all the known first integrals.

On top of total energy, the magnetohydrodynamic equations conserve two more invariants, that is, two kinds of helicities. One is magnetic helicity,

$$
\int \mathcal{A} \cdot \boldsymbol{B} d \boldsymbol{x}
$$

and the other one is cross helicity,

$$
\int \boldsymbol{u} \cdot \boldsymbol{B d x}
$$

To each helicity conservation there corresponds respective Weber transform. Weber transform for magnetic helicity is well-known and straightforward. To see this, it suffices to write down the equations for vector potentials $\boldsymbol{A}$ for the magnetic field,

$$
\frac{D \mathcal{A}}{D t}=-\mathcal{A}(\nabla \boldsymbol{u})^{T}-\nabla \chi
$$

It follows that the corresponding Weber transform takes the following form:

$$
\mathcal{A}=\boldsymbol{P}\left[\mathcal{A}_{0}(\nabla \boldsymbol{a})^{T}\right],
$$

where $\boldsymbol{P}$ is solenoidal projection.

The lesser-known Weber transform for cross helicity is due to Refs. 10 and 11 (see also Ref. 12). We briefly describe it following the treatment of Ref. 11. We introduce $\boldsymbol{m}$ by

$$
\begin{aligned}
& \frac{D \boldsymbol{m}}{D t}=\boldsymbol{m} \cdot \nabla \boldsymbol{u}+\boldsymbol{J}, \\
& \nabla \cdot \boldsymbol{m}=0 .
\end{aligned}
$$

It follows from this definition that

$$
\frac{D(\boldsymbol{m} \cdot \delta \mathbf{S})}{D t}=\boldsymbol{J} \cdot \delta \mathbf{S}
$$

thus $\boldsymbol{m}$ has a meaning of a current flux across unit area per unit time.

If we define a generalized velocity by

$$
\widetilde{\boldsymbol{u}} \equiv \boldsymbol{u}+\boldsymbol{B} \times \boldsymbol{m},
$$

then after some manipulations we obtain

$$
\frac{D}{D t}\left(\widetilde{\boldsymbol{u}} \cdot \frac{\partial \boldsymbol{x}}{\partial a_{i}}\right)=\frac{\partial}{\partial a_{i}}\left(\frac{|\boldsymbol{u}|^{2}}{2}-p\right) .
$$

It is readily verified that

$$
\boldsymbol{u}=\boldsymbol{P}\left[\widetilde{\boldsymbol{u}}_{0}(\boldsymbol{a})(\nabla \boldsymbol{a})^{T}+\boldsymbol{m} \times \boldsymbol{B}\right] .
$$

The fact that $\tilde{\boldsymbol{u}}$ enjoys Weber transform implies that corresponding generalized vorticity defined by

$$
\widetilde{\boldsymbol{\omega}}=\nabla \times \widetilde{\boldsymbol{u}}
$$

satisfies

$$
\frac{D \widetilde{\boldsymbol{\omega}}}{D t}=\widetilde{\boldsymbol{\omega}} \cdot \nabla \boldsymbol{u} .
$$

Using this, we are going to show that if the magnetic field $\boldsymbol{B}$ is smooth with respect to $\boldsymbol{a}$, then so is the velocity $\boldsymbol{u} .^{13} \mathrm{In}$ view of (2) we find that $\partial x_{i} / \partial a_{j}$ should be smooth except for a slim possibility that the dot product cancels singular contributions. Then, by

$$
\widetilde{\boldsymbol{\omega}}=\widetilde{\boldsymbol{\omega}}_{0} \cdot \frac{\partial}{\partial \boldsymbol{a}} \boldsymbol{x}(\boldsymbol{a}, t),
$$

we find that $\tilde{\boldsymbol{\omega}}$ is also smooth.

We consider each term on the right-hand side of

$$
\boldsymbol{\omega}=\tilde{\boldsymbol{\omega}}-\nabla \times(\boldsymbol{B} \times \boldsymbol{m}) .
$$


Equation (3) can be solved by a method of variation of constant as

$$
m_{i}(t)=\left(m_{j}(0)+\int_{0}^{t} J_{k}\left(t^{\prime}\right) \frac{\partial a_{i}}{\partial x_{k}} d t^{\prime}\right) \frac{\partial x_{i}}{\partial a_{j}} .
$$

By

$$
\frac{\partial}{\partial x_{i}}=\frac{\partial a_{j}}{\partial x_{i}} \frac{\partial}{\partial a_{j}}
$$

smoothness with respect to $\boldsymbol{a}$ implies smoothness with respect to $\boldsymbol{x}$. Then, $\boldsymbol{J}$ is also smooth since $\boldsymbol{J}=\nabla \times \boldsymbol{B}$. Because of the incompressibility condition

$$
\frac{\partial\left(x_{1}, x_{2}, x_{3}\right)}{\partial\left(a_{1}, a_{2}, a_{3}\right)}=1(\neq 0),
$$

the Jacobian matrix $\partial x_{i} / \partial a_{j}$ is invertible and the inverse matrix is also smooth. We see that $\boldsymbol{m}$ is smooth since each term in (5) is smooth. Therefore, each term in (4) is smooth, and we deduce that vorticity remains smooth.

We have seen that as long as the magnetic field is smooth, no singularity can show up in the vorticity field. This suggests that we need to monitor growth of the magnetic field in numerical studies purporting singularity forma- tion. In Ref. 9, while the condition is imposed both on the velocity and the magnetic fields, it concerns only their first derivatives, that is, the vorticity field and the current density. Here, our assumption is made only on the magnetic field, but higher derivatives are required. It may be of interest to try to obtain a functional analytic bound by dealing with the magnetic field only.

${ }^{1}$ R. M. Kerr and A. Brandenburg, Phys. Rev. Lett. 83, 1155 (1999).

${ }^{2}$ I. Klapper, Phys. Plasmas 3, 4281 (1996).

${ }^{3}$ D. Cordoba and C. Fefferman, SIAM J. Math. Anal. 33, 786 (2001).

${ }^{4}$ I. Klapper, Phys. Plasmas 5, 910 (1998).

${ }^{5}$ D. Cordoba and C. Marliani, Commun. Pure Appl. Math. 53, 512 (2000).

${ }^{6}$ J. T. Beale, T. Kato, and A. Majda, Commun. Math. Phys. 94, 61 (1984).

${ }^{7}$ W. E and Ch.-W. Shu, Phys. Fluids 6, 49 (1994).

${ }^{8}$ D. Chae and H.-S. Nam, Proc. - R. Soc. Edinburgh, Sect. A: Math. 127, 935 (1997).

${ }^{9}$ R. E. Caflisch, I. Klapper, and G. Steele, Commun. Math. Phys. 184, 443 (1997).

${ }^{10}$ V. E. Zakharov and E. A. Kuznetsov, Dokl. Akad. Nauk SSSR 194, 1288 (1970) [Sov. Phys. Dokl. 15, 913 (1971)].

${ }^{11}$ V. A. Vladimirov and K. H. Moffatt, J. Fluid Mech. 283, 125 (1995).

${ }^{12}$ E. A. Kuznetsov and V. P. Ruban, Phys. Rev. E 61, 831 (2000).

${ }^{13}$ If we assume that $\boldsymbol{B}$, is smooth with respect to both $\boldsymbol{a}$ and $t$, then smoothness of $\boldsymbol{u}$ follows immediately from $\boldsymbol{F}^{-1} D \boldsymbol{F} / D t=\nabla \boldsymbol{u}$, where $F_{i j}=\partial x_{i} / \partial a_{j}$ is the Jacobian matrix. 